\title{
Twisted Ovarian filbroma Mimicking as an Ectopic Pregnancy
}

Aruna Nigam, Shweta Jain, Prabha Lal

From the Department of Obstetrics and Gynaecology, Lady Hardinge

Medical College, New Delhi-1 10001, India

Abstract:

Ovarian fibromas are benign tumors arising from the stromal component of the ovary. They mostly are asymptomatic. These tumors are often detected as incidental finding detected on routine gynaecological examination, transvaginal ultrasound, computer tomography or magnetic resonance imaging. We report a case of twisted ovarian fibroma with raised HCG levels and ascites mimicking ruptured ectopic pregnancy in young nulliparous married female.

Key words: Fibroma, Ectopic Pregnancy, Ascites, Ovarian Neoplasms, Pregnancy.

\section{Introduction}

Ovarian fibromas are most common benign solid tumors of the ovary and accounts for approximately $1-4 \%$ of all benign ovarian tumors [1]. They are generally asymptomatic and typically detected in middle aged women on palpation during routine gynecological examination. A rare case of twisted ovarian fibroma with raised HCG levels and ascites mimicking ruptured ectopic pregnancy in young nulliparous married female is being reported.

\section{Case Report}

A 22 year old nulliparous married female presented in emergency with acute pain in lower abdomen and vaginal bleeding for one day following one and a half month amenorrhea. History of a fainting attack was present. There were no associated bladder or bowel complaints. On general examination, her pulse rate was $110 / \mathrm{min}$ and blood pressure was $96 / 60 \mathrm{~mm} \mathrm{Hg}$. Abdominal examination revealed tenderness in left iliac fossa. Vaginal examination revealed a normal sized uterus and a large irregular tender mass of $5 \times 10 \mathrm{~cm}$ size, cystic to firm in consistency. Cervical motion tenderness was present. Hematological investigations were normal with hemoglobin of $10 \mathrm{gm} / \mathrm{dL}$ and TLC of $5600 / \mathrm{mm}^{3}$. Ultrasound showed a mixed echogenic mass of $9.5 \times 4.5 \mathrm{~cm}$ in left adnexa which was extending posterior to the uterus. Uterus was normal sized with endometrial thickness of $7 \mathrm{~mm}$. Free fluid was present in the pelvis. Her urine pregnancy test was positive. A diagnosis of ruptured ectopic pregnancy was entertained and patient was undertaken for emergency laparotomy. Peroperatively,

\section{Corresponding Author: Dr. Aruna Nigam}

Email: prakasharuna@hotmail.com

Received: January 4, 2013 | Accepted: January 29, 2013 | Published Online: February 10, 2013

This is an Open Access article distributed under the terms of the Creative Commons Attribution License (creativecommons.org/licenses/by/3.0)

Conflict of interest: None declared | Source of funding: Nil | DOI: http://dx.doi.org/10.17659/01.2013.0016 
100 c.c. of serous fluid was present in the abdominal cavity. A big, white mass measuring $8 \times 6 \times 4 \mathrm{~cm}$ with irregular surface was present posterior to the uterus which was attached to the left ovary with a very thin pedicle measuring $0.5 \mathrm{~cm}$ in width and around $1.5 \mathrm{~cm}$ long and twisted thrice [Fig. 1]. Both the tubes and ovaries were normal. No evidence of any other ectopic mass was found. Mass was adherent to the sigmoid colon posteriorly. The mass was removed after releasing the adhesions and cutting it from the pedicle. Curettage of endometrial cavity was done simultaneously. Cut surface revealed firm grey white colour with yellowish area in between [Fig.2]. There were no areas of hemorrhage and necrosis. Histopathological examination revealed cells arranged compactly in intersecting fascicles and vague storiform pattern [Fig.3]. The cells were elongated, spindle shaped with indistinct cell boundary, eosinophillic fibrillary cytoplasm, elongated nuclei with pointed ends showing fine chromatin and inconspicuous nucleoli [Fig.4]. No mitotic figures were seen. Special stain for Masson's trichrome was negative and immunohistochemistry for desmin was also negative. Histological features were consistent with ovarian fibroma. Endometrial curetting's revealed broken up bits of secretory endometrial glands with oedematousstroma infiltrated by acute and chronic inflammatory cells. Although urine pregnancy test was positive pre-operatively, to confirm elevated HCG levels, a serum sample was sent in the immediate post-operative period and value obtained was $125 \mathrm{mlU} / \mathrm{mL}$. Beta HCG resolved to less than $2 \mathrm{mIU} / \mathrm{mL}$ after 2 weeks. Her post-operative course was uneventful. Patient has been on follow-up for last 1 year and have conceived recently.

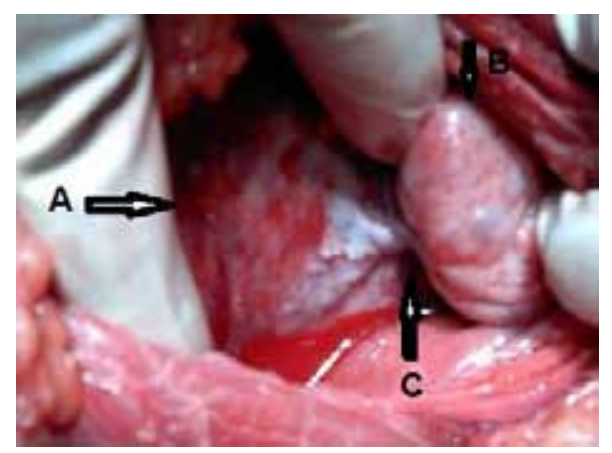

Fig.1: Intraoperative picture showing $[A]$ fibroma attached to the $[B]$ ovary with $[C]$ twisted pedicle.

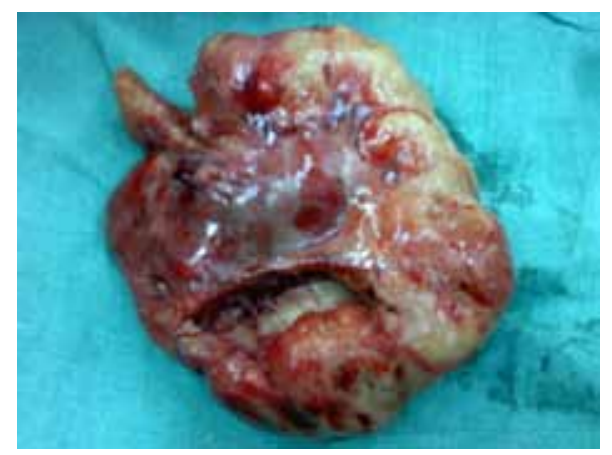

Fig.2: Cut section of the ovarian fibroma. 


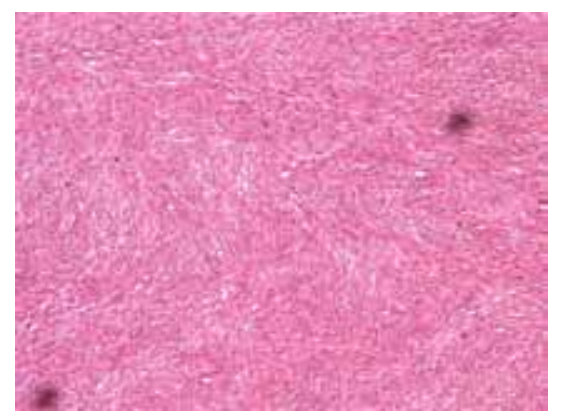

Fig.3: Histopathology of ovarian fibroma showing compactly arranged cells in intersecting fascicles and vague storiform pattern under low magnification (10x).

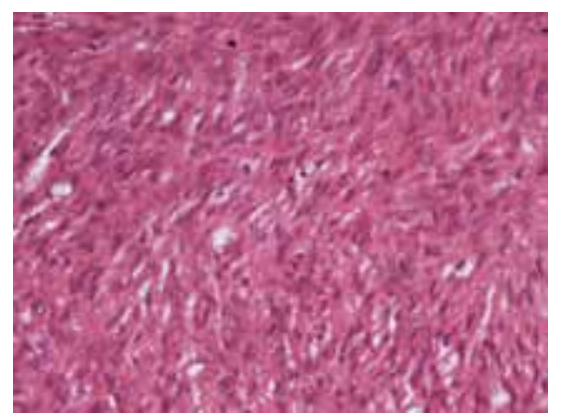

Fig.4: Elongated spindle shaped cells with indistinct cell boundry, osinohillicfibrillary cytoplasm, elongated nuclei, inconspicuous nucleoli under high magnification (40x).

\section{Discussion}

One of the purposes of presenting this case is the diagnostic dilemma as none of the symptoms or investigations pointed towards the ovarian mass. The typical triad of amenorrhoea followed by pain and bleeding with positive urine pregnancy test and ultrasonography finding of adnexal mass with free fluid in pelvis indicated towards ruptured ectopic pregnancy. Although elevated CA-125 levels have been found with this tumor, but elevated beta-HCG levels in association with ovarian fibroma has yet not been observed, thus raised beta HCG level in the present case can be attributed to chemical pregnancy.

Ovarian fibroma is a rare benign tumor originating from the connective tissue of the ovarian cortex. According to the WHO classification of ovarian neoplasm, they represent a subgroup of granulose-theca cells tumors and belong to theca fibroma group. The fibroma arises from spindle cells which produces collagen. It is the commonest subtype of the sex cord-stromal tumors [2]. The mean age at diagnosis is 48 years and $90 \%$ of patients are at least 30 years old when they are diagnosed with fibroma [3]. In our case patient was 22 years old. These tumors are often asymptomatic despite their large size and are mostly discovered on routine physical examination. Occasionally large fibromas may develop a pedicle and undergo torsion causing acute abdominal symptoms as in the present case. Ascites or classic Meig's syndrome with additional hydrothorax has been found in $10 \%$ of all cases and $40 \%$ of tumors larger than $10 \mathrm{~cm}$ in maximum diameter [3]. Development of 
ascites is attributed to inefficient lymphatic drainage through a small-for-size pedicle and a lack of a real tumor capsule to prevent leakage of lymph in the peritoneal cavity. Hydrothorax is secondary to ascites due to trans diaphragmatic passage. Treatment is always surgical because of the low probability of malignancy. Leung SW et al reviewed 23 cases of ovarian fibroma and concluded that surgical removal of these solid ovarian tumors is recommended [4]. Open or laparscopic approaches are both acceptable but careful handling of the tumor is mandatory as approximately $1 \%$ of these are malignant. Ultrasound appearance of the tumor is variable, most manifest as solid hypo-echoic masses with sound attenuation. Lack of tumor enhancement on computed tomography is the most reliable sign of ovarian torsion and should facilitate prompt surgical intervention [5].

\section{References}

1. Chechia A, Attia L, Temime RB, Makhlouf T, Koubaa A. Incidence, clinical analysis and management of ovarian fibromas and fibrothecomas. Am J Obstet Gynecol. 2008; 199:473.e 1-4.

2. Paladini D, Testa A, Von Holsbeke C, Mancari R, Timmerman D, Valentin L. Imaging in gynecological disease: clinical and ultrasound characteristics in fibroma and fibrothecoma of the ovary. Ultrasound Ostet Gynecol. 2009;34:188-195.

3. Sivanesaratnam V, Dutta R, Jayalakshmi P. Ovarian fibroma-clinical and histopathological characteristics. Int J Gynaecol Obstet.1990;33:243-247.

4. Leung SW, Yuen PM. Ovarian Fibroma: A review on the clinical characteristic, diagnostic difficulties and management options of 23 cases. Gynecol Obstet Invest. 2006;62:1-6.

5. Mak CW, Tzeng WS, Chen CY. Computed tomography appearance of ovarian fibro-thecomas with and without torsion. Acta Radiol. 2009;50:570-575. 